\title{
Melatonin enhances therapeutic efficacy of cardiac progenitor cells for myocardial infarction via targeting miR-98/STAT3 signal
}

\author{
Benzhi Cai ${ }^{1,2}$, Wenya Ma ${ }^{1,2}$, Fengzhi Ding ${ }^{1}$, Lai Zhang ${ }^{1}$, Fan Yang ${ }^{1}$, Ye Yuan ${ }^{1,2}$, Yanjie Lu ${ }^{1}$, \\ Zhimin $\mathrm{Du}^{1,2}$
}

${ }^{I}$ Pharmacy, Department of Pharmacy of The Second Affiliated Hospital, Harbin Medical University (the Heilongjiang Provincial Key Laboratory of Drug Research, Harbin Medical University), Harbin, China; Department of Pharmacology (Key Laboratory of Cardiovascular Research, Ministry of Education), College of Pharmacy, Harbin Medical University, Harbin, China, ${ }^{2}$ Department of Clinical Pharmacy of The Second Affiliated Hospital, Harbin Medical University, Harbin, China

Melatonin, the hormone produced by pineal gland plays an important role in the regulation of circadian rhythms, and also processes many biological activities such as antioxidant, cancer protection and insomnia. Cardiac progenitor cells (CPCs) have emerged as a promising therapeutic strategy for myocardial infarction (MI). However, the low survival of transplanted CPCs in infarcted myocardium limits the successful use in treating MI. In the present study, we aimed to investigate if melatonin may protect against oxidative stress-induced CPCs damage and enhance its therapeutic efficacy for MI. Here, we found that H2O2-induced the proliferation reduction and apoptosis of c-kit+ CPCs was remarkably attenuated by melatonin treatment in vitro, and the combination of CPCs and melatonin significantly improved the functions of post-infarct hearts compared with CPCs or melatonin alone in vivo. Interestingly, we found that melatonin was capable to inhibit the increase of miR-98 level by H2O2 in CPCs. The proliferation reduction and apoptosis of CPCs induced by $\mathrm{H} 2 \mathrm{O} 2$ was aggravated by miR-98 overexpression but abrogated by the knockdown of miR-98. In vivo, transplantation of CPCs with miR-98 silencing caused the more significant improvement of cardiac functions in MI than CPCs. We further found that miR-98 overexpression suppressed The signal transducer and activator of the transcription 3 (STAT3) expression by targeting at its mRNA, and thus aggravated $\mathrm{H} 2 \mathrm{O} 2$-induced the reduction of Bcl-2 protein. Finally, overexpression of miR-98 abrogated the antiapoptotic effects of melatonin on CPCs. In summary, our study revealed that melatonin protected c-kit+ CPCs against oxidative stress-induced damage via miR-98/STAT3 pathway, which provides one new strategy to improve CPC-based therapy for MI. 